九州大学学術情報リポジトリ

Kyushu University Institutional Repository

\title{
Development of a Detection Method for Adulterated Onion Powder using Raman Spectroscopy
}

Lee, Sangdae

Convergence components \& agricultural machinery application center, Korea Institute of Industrial Technology

Lohumi, Santosh

Department of Biosystems Machinery Engineering, Chungnam National University

Lim, Hyoun-Sub

Department of Applied Biology, Chungnam National University

Gotoh, Takafumi

Kuju Agricultural Research Center, Faculty of Agriculture, Kyushu University

他

https://doi.org/10.5109/1526312

出版情報：九州大学大学院農学研究院紀要. 60 (1)，pp.151-156，2015-02-27. Faculty of Agriculture, Kyushu University

バージョン :

権利関係 : 


\title{
Development of a Detection Method for Adulterated Onion Powder using Raman Spectroscopy
}

\author{
Sangdae LEE ${ }^{1}$, Santosh LOHUMI ${ }^{2}$, Hyoun-Sub LIM ${ }^{3}$, Takafumi GOTOH ${ }^{4}$, \\ Byoung-Kwan $\mathrm{CHO}^{2 *}$, Moon S. KIM ${ }^{5}$ and Soo-Hee $\mathrm{LEE}^{6}$
}

\author{
Kuju Agricultural Research Center, Kyushu University, \\ Kuju 4045-4, Taketa City, 878-020, Oita, Japan \\ (Received October 31, 2014 and accepted November 14, 2014)
}

\begin{abstract}
The purpose of this study was to develop a detection technique for adulterated powder products using Raman spectroscopy. While conventional methods for food quality analysis require time and pretreatment procedures, Raman spectroscopy can be used to detect a food component rapidly and nondestructively without pretreatment. In this study, Raman spectra were measured using a $785 \mathrm{~nm}$ laser source in the range 3,200 170 $\mathrm{cm}^{-1}$ and multivariate analytical method using partial least square regression (PLSR) was applied to develop optimal models for predicting the mixed cornstarch concentration in adulterated onion powders. A range of preprocessing methods were applied to correct Raman spectra by reducing the systematic noise and variations produced by the light source and light scattering of the sample surface. Raman data were predicted with a high $\mathrm{R}^{2}$ value and low standard error for the entire preprocessing data set. The method proved its effectiveness to rapidly detect adulteration in powdered food.
\end{abstract}

Key words: cornstarch, onion powder, adulteration, Raman spectroscopy, PLSR

\section{INTRODUCTION}

Food adulteration is the contamination of food with chemical or physical substances that should not be present in food. Adulteration of food products for economic gain with cheap ground or chemical materials could pose serious health threats to consumers. Food products have long and complicated processing and supply chains, which make the products vulnerable to adulteration. Thus, authentication is important, both for official bodies in charge of labeling and for industries. Adulteration of a wide variety of food products including milk and milk products, fruit juice, alcohol, coffee, flour, meat products, and even fruits is on the rise (Lakshmi, 2012). Powdered food products, particularly spices, are one of the most susceptible food stuffs prone to adulteration. However, many adulterated spices are still circulating in the market, causing consumers to worry about food safety. In 2013, Korean government decided that extermination of adulterated food products should be a high priority. In line with this goal, the Ministry of Food and

Convergence components \& agricultural machinery application center, Korea Institute of Industrial Technology, 119, Jipyeongseonsandan 3-gil, Baeksan-myeon, Gimje-si, Jeollabuk-do, 576-881, South Korea

${ }^{2}$ Department of Biosystems Machinery Engineering, Chungnam National University, 220 Gung-dong, Yuseong-gu, Daejeon, 305-764, South Korea

${ }^{3}$ Department of Applied Biology, Chungnam National University, 220 Gung-dong, Yuseong-gu, Daejeon, 305-764, South Korea

${ }^{4}$ Kuju Agricultural Research Center, Faculty of Agriculture, Kyushu University, Kuju, Oita, Japan

${ }^{5}$ Environmental Microbial and Food Safety Laboratory, USDAARS, Beltsville, MD 20705, USA

${ }^{6}$ Life \& Technology co. LtD, 53 Jeongnamdong-ro, Jeongnammyeon, Hwaseong-si, 445-964, South Korea

* Corresponding Author (E-mail: chobk@cnu.ac.kr)
Drug Safety prepared a guideline to distinguish the raw materials of food products using DNA analysis methods and they make efforts to eradicate the adulterated foods.

In food quality control analyses, principally the determination of adulteration or authenticity involves the use of a range of conventional chemical methods, and various devices and procedures such as chromatography, thermal analysis, microscopic analysis and other biotechnological methods. Chromatographic methods such as high-performance liquid chromatography (HPLC) and gas-liquid chromatography (GLC) have been successfully applied to determine food quality of a variety of food products but its destructive nature and high analytical cost limited its applications for routine use.

Spectroscopic techniques have been shown to have the potential for applications in quality analysis in food science, as well as pharmaceutical and petrochemical industries (Baeten \& Dardenne, 2002). Various types of spectroscopic techniques, such as near infrared (NIR), Fourier transform infrared (FT-IR), Raman spectroscopy, and hyperspectral imaging (HSI), are available for quality analysis of different types of agro-food products. For example, these spectroscopic techniques have been applied for the analysis of food authenticity, including detection of melamine adulteration in milk powder and in soya bean meal using NIR spectroscopy (Lu et al., 2009; Haughey et al., 2012). A large number of studies were carried out to determine sugar content and adulteration in fruit juice using FT-IR spectroscopy (Jha \& Gunasekaran, 2010). In addition, Wijaya et al. (2014) used Raman spectroscopy for the detection of the neonicotinoid pesticide acetamiprid in foods, and melamine adulteration has been detected in milk powder using HSI (Fu et al., 2014).

Raman spectroscopy is a vibrational spectroscopy technique used to collect a unique chemical fingerprint 
of molecules. Each molecule has a different set of vibrational energy levels, and the photons have unique wavelength shifts. Raman spectra show different peaks corresponding to unique Raman excitations. Raman and infrared spectroscopies are complimentary techniques for food and feedstuff analysis. The main advantages of Raman spectroscopy over NIR and MIR spectroscopy are as follows: (i) there is no or minimum interference from water, (ii) aqueous solutions can be analyzed without or with minimal interference of water, (iii) inorganic materials are more easily analyzed, and (iv) samples can be analyzed through glass or polymer packaging. Raman spectroscopy gives an objective and accurate result. Kizil et al. used Fourier transform infrared (FT-IR) and FT-Raman methods for rapid characterization and classification of selected irradiated starch samples and detected biochemical changes due to irradiation. Lee et al. developed an optimal PLSR model for determining the protein and oil contents of soybeans using a dispersive Raman spectroscopy method. Finally, Qin et al. (2010) demonstrated the potential of Raman spectroscopy in combination with hyperspectral imaging for the detection of melamine adulteration in milk powder, and suggested that this may be an effective method for detection of adulterated foods. Thus, Raman spectroscopy has been shown to have a variety of applications within this field.

The objective of this study was to detect cornstarch adulteration in onion powder using Raman spectroscopy. PLSR was applied to preprocessed Raman spectral data of pure onion powder and seven different concentrations of starch within adulterated onion powder.

\section{MATERIALS AND METHODS}

\section{SAMPLE PREPARATION}

Onion powder and cornstarch with 100\% purity were obtained from a local market in Korea. Cornstarch was used as the adulterant in the onion powder. The samples were stored at room temperature until use. Weights of dried onion powder and cornstarch were measured with an electronic balance (Model AR2130, Ohaus Corp., NJ, USA). The cornstarch was then mixed with the onion powder to final concentrations (w/w) of 5\%, 10\%, 15\%, $20 \%, 25 \%, 30 \%$, and $35 \%$. One group of pure onion powder also prepared. Thus, a total of 80 samples (10 samples for each group) were prepared. Each combination was manually mixed and then transferred to a $50 \mathrm{~mL}$ conical type tube. Further mixing was accomplished by placing the filled tubes onto a high speed shaker (Model G560, Scientific Industries, Inc., NY, USA). Each of 10 sample holders with $10 \mathrm{~mm}$ in diameter and $3 \mathrm{~mm}$ in thickness were filled with pure and adulterated powders and made a flat level surface for Raman measurement.

\section{SPECTROSCOPIC MEASUREMENTS}

Raman scattering signal of the pure and adulterated onion powders were obtained using i-Raman plus (B\&W Tek Inc., USA). A $300 \mathrm{~mW}$ laser source at $785 \mathrm{~nm}$ excitation wavelength was used to minimize the noise genera- tion and fluorescence signal from the samples. All Raman spectral data of powder samples were collected in a darkroom to exclude the noise signal created by unnecessary external lighting. A BAC100 model from B\&W Tek Inc. was used as a standard probe. For optimal Raman spectra, 100\% laser power and $6 \mathrm{~s}$ of exposure time were set. The Raman instrument measured the reflected Raman scattering signal, and a total of 16 successive scans from each sample were collected over the wavelength range between 3,200 and $170 \mathrm{~cm}^{-1}$ at $4.5 \mathrm{~cm}^{-1}$ intervals. Averaged spectra were used for developing a PLSR model. Fig. 1 shows the Raman spectroscopy system and the inside view of the darkroom.

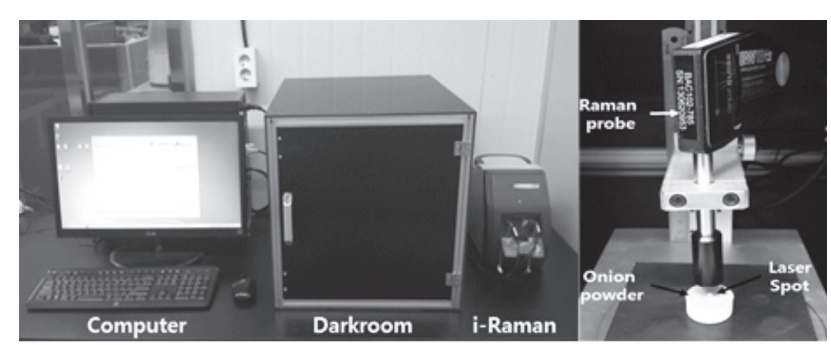

Fig. 1. Raman spectroscopy system for detection of the adulterated onion powder.

\section{SPECTRAL DATA TREATMENT AND ANALYSIS}

Because a Raman scattering signal was generated by using a strong laser light source, agricultural products may emit strong fluorescence signals that mask the characteristic Raman scattering signal of the sample. In this study, a simple and efficient polynomial curve fitting method was employed to remove the fluorescence effect of the onion powder sample and to correct the base-line of the obtained Raman spectra. Polynomial curve fitting involves determining the proper order polynomial for obtaining a baseline through iterative calculation. Various orders of polynomial such as first, second, fourth, and sixth were tested for obtaining the optimal Raman spectra from pure and adulterated onion powder samples. Ultimately, a second order polynomial equation was employed for the fluorescence and baseline correction of spectral data of onion powder because this polynomial provided the best prediction compared with the other polynomials.

Direct analysis of spectroscopic data is generally not possible because spectral data are often affected by a variety of factors, such as base-line shift, instrumental drifts and difference in particle size. Thus, pretreatment is needed to reduce unwanted interference without interfering with existing useful information and to improve the subsequent multivariate regression, classification model, or exploratory analysis (Rinnan et al., 2009). Thus, seven preprocessing methods, such as mean normalization, maximum normalization, range normalization, multiplicative scatter correction (MSC), standard normal variate (SNV), Savitzky-Golay $1^{\text {st }}$ derivative, and Savitzky-Golay $2^{\text {nd }}$ derivative methods were applied to the corrected Raman spectra. The preprocessed spectra were used to develop an optimal PLSR model described 
for prediction of the adulteration level in the pure and adulterated onion powder samples. In order to determine the optimal model among the developed PLSR models, $R_{c}^{2}$ (coefficient of determination in calibration), SEC (standard error of calibration), $R_{v}^{2}$ (coefficient of determination in validation), SEV (standard error of validation), $R_{p}^{2}$ (coefficient of determination in prediction), SEP (standard error of prediction), and F (latent variables) values were compared.

The data for 80 samples were split into two sets: a calibration set consisting of 56 samples, and a prediction set consisting of 24 samples. The PLSR models were built with the calibration set using a full cross-validation method. This is the method generally used in developing PLSR models for detecting a specific material with a spectral dataset. PLSR is a supervised classification method and performs well in cases with many variables. PLSR has been used in food authentication studies based on spectroscopic data. Multivariate analysis was performed using MATLAB software version 7.0.4 (The Mathworks, Nitick, MA, USA).

\section{RESULTS AND DISCUSSION}

\section{RAMAN SPECTRUM}

Fig. 2 shows the original Raman spectrum of pure cornstarch. According to the food and nutrient data system of the Korean Ministry of Food and Drug Safety, cornstarch (g/100 g) includes $78.1 \mathrm{~g}$ carbohydrate, 10.1 $\mathrm{g}$ protein, and $3.9 \mathrm{~g}$ fat. Raman spectra of cornstarch mainly originate from its carbohydrate, protein, and fat components. A strong Raman band at $481 \mathrm{~cm}^{-1}$ is one of the dominating and important skeletal vibration modes of the pyranose ring in polysaccharides (amylose, amylopectin, cellulose, and starch). Raman spectra of polysaccharides in the region between 800 and $1500 \mathrm{~cm}^{-1}$ originate from the vibrational state of its monomer glucose. The Raman band at $870 \mathrm{~cm}^{-1}$ is attributed to the $\mathrm{C}-\mathrm{H}$, $\mathrm{CH}_{2}$ deformation, while the Raman band in the vicinity of $920-960 \mathrm{~cm}^{-1}$ is attributed to the skeletal mode vibrations of the $\alpha-1,4$ glycosidic linkage. In this study, the Raman band at $947 \mathrm{~cm}^{-1}$ is attributed to the glycosidic linkages in cornstarch. Raman bands at 1131, 1341, and $1460 \mathrm{~cm}^{-1}$ are attributed to $\mathrm{C}-\mathrm{O}$ stretching and $\mathrm{C}-\mathrm{O}-\mathrm{H}$ bending, $\mathrm{C}-\mathrm{O}-\mathrm{H}$ bending and $\mathrm{CH}_{2}$ twisting, and $\mathrm{CH}_{2}$ bending, respectively.

Fig. 3 shows the original Raman spectra from 80 pure and adulterated onion powder samples. There was a strong intensity variation due to the fluorescence signal of the onion powder sample. A second order polynomial equation was used to remove the background noise from the fluorescence signal. Fig. 4 shows the Raman spectra after removal of the fluorescence signal from original onion Raman spectra and Raman bands representing various constituents of onion powder were identified. Savitzky-Golay 1st derivative-preprocessed spectra are shown in Fig. 5. The Savitzky-Golay method is a widely used preprocessing technique that can effectively eliminate different types of noise, such as base-line drift, tilt, and reverse. Strong Raman bands at 420 and $552 \mathrm{~cm}^{-1}$ are attributed to the $\mathrm{C}-\mathrm{C}-\mathrm{C}$ bending and $\mathrm{C}-\mathrm{C}-\mathrm{O}$ bending of glucose rings. The Raman band at $947 \mathrm{~cm}^{-1}$ is attributed to the skeletal mode vibrations of the $\alpha-1,4$ glycosidic linkage. Raman bands at 1366 and $1472 \mathrm{~cm}^{-1}$ are attributed to $\mathrm{CH}_{2}$ bending and skeletal vibrations. The onion powder (g/100 g) included $79.8 \mathrm{~g}$ carbohydrate, $8.8 \mathrm{~g}$

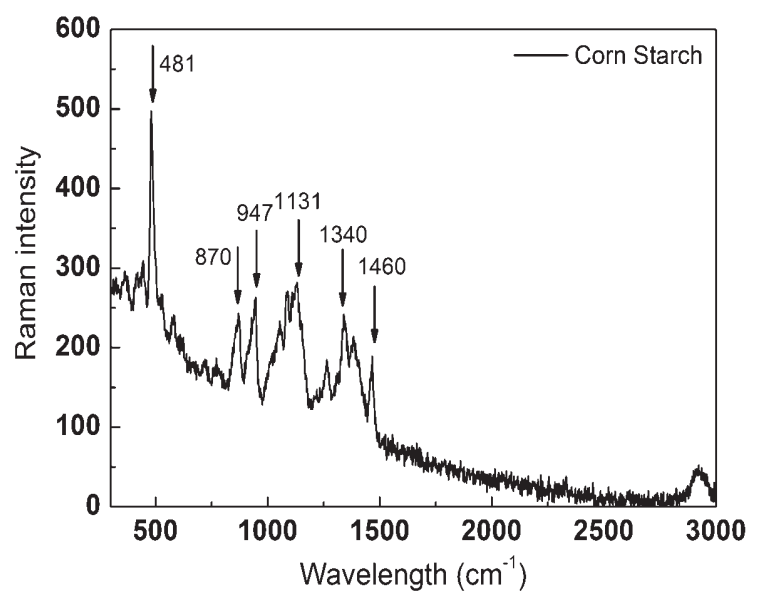

Fig. 2. Raman spectrum of cornstarch.

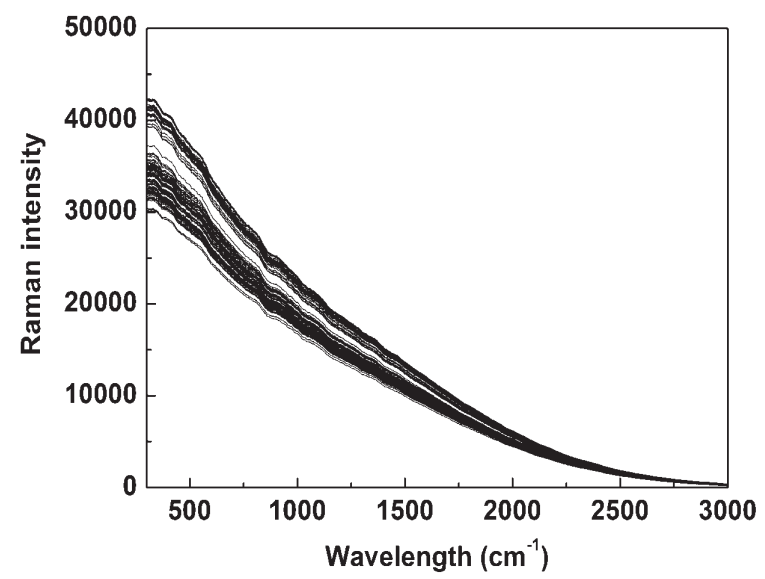

Fig. 3. Original Raman spectra of pure and adulterated onion powder samples.

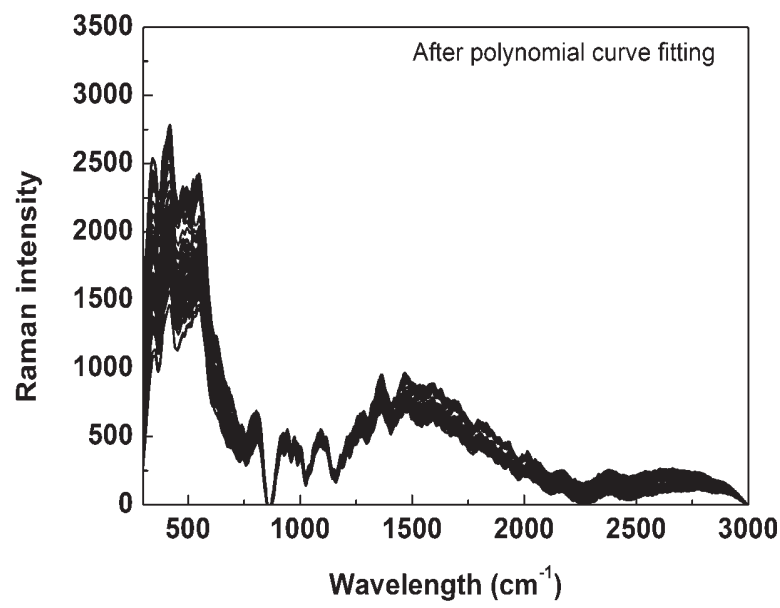

Fig. 4. Raman spectra modified with polynomial curve fitting method. 
protein, and $1.1 \mathrm{~g}$ fat. The components of cornstarch and onion powder were very similar with different only in concentrations.

\section{PLSR MODEL}

The major band of the Raman spectrum in Fig. 5

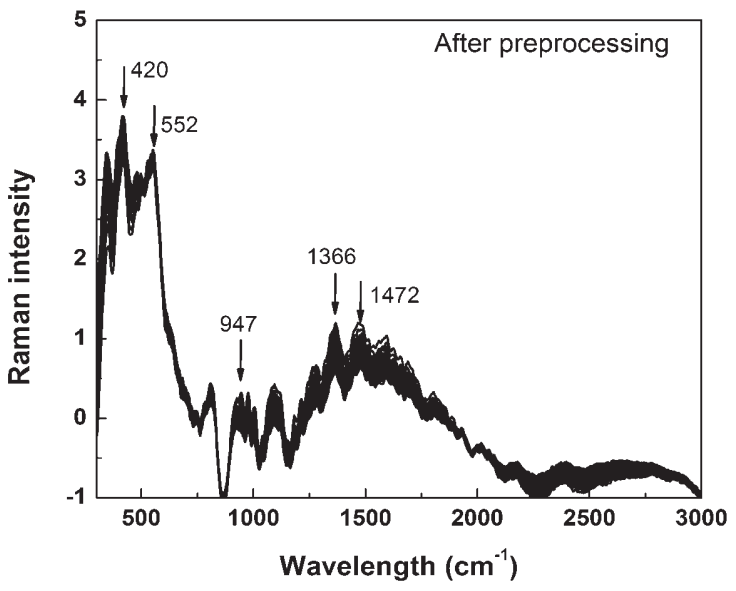

Fig. 5. Raman spectra modified with Savitzky-Golay $1^{\text {st }}$ derivative preprocessing.

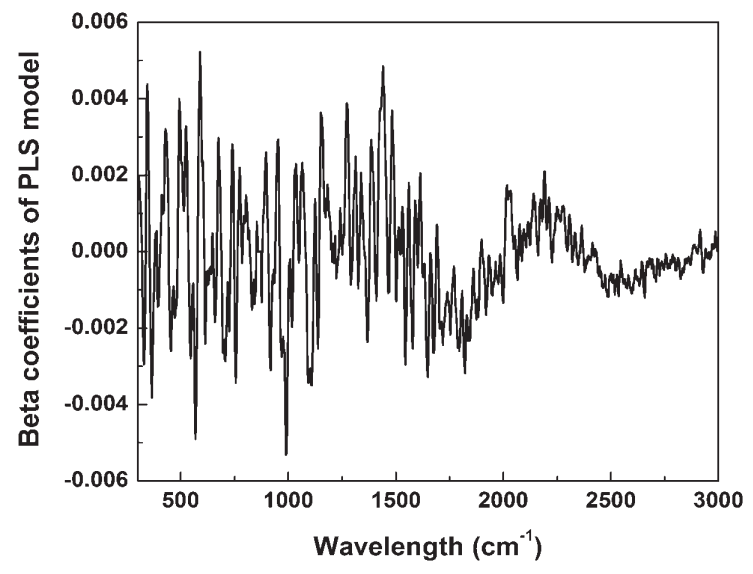

Fig. 6. Beta coefficient values of PLSR model. occurred between 2,000 and $300 \mathrm{~cm}^{-1}$, which were consistent with the Raman band information reported for cornstarch in the literature (Almeida et al., 2010). Difference in the spectral features of pure and adulterated onion powder were minor and could not be visually discerned. Thus, a multivariate analytical method of PLSR was employed to evaluate any spectral variations between pure and adulterated onion powder samples because analysis of multiple bands has been shown to be effective in quantifying the cornstarch content of adulterated onion powder. PLSR is an effective dimensional reduction method for Raman spectral data analysis. It is a widely used method of spectral modeling integrating principal component analysis and multiple linear regression. This method provides information on the dependent variable and simultaneously reduces the dimension of the spectral matrix. Preprocessed spectra were used to develop PLSR models to predict the mixed cornstarch concentration in adulterated powder samples. To evaluate the optimal PLSR model, the $R_{c}^{2}$, SEC, $R_{v}^{2}, \mathrm{SEV}, R_{p}^{2}$, SEP, and F of the PLSR model were determined for each preprocessed spectral data set. The results of PLSR models with each preprocessing method are shown in Table

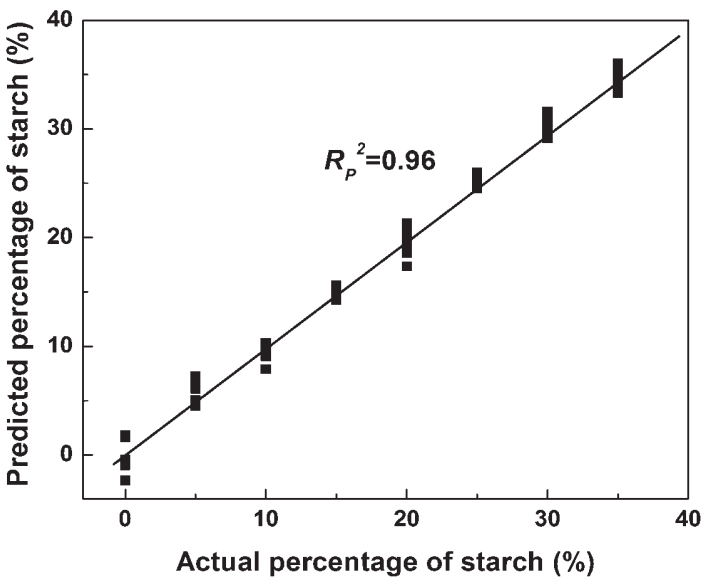

Fig. 7. Actual percentages and predicted percentages of cornstarch using Savitzky-Golay $1^{\text {st }}$ derivative-preprocessed PLSR model

Table 1. Performance of PLSR models developed using preprocessed Raman spectra

\begin{tabular}{|c|c|c|c|c|c|c|c|}
\hline \multirow{2}{*}{ preprocessing } & \multicolumn{2}{|c|}{ Calibration } & \multicolumn{2}{|c|}{ Validation } & \multicolumn{2}{|c|}{ Prediction } & \multirow{2}{*}{$\mathrm{F}$} \\
\hline & $R_{c}^{2}$ & SEC (\%) & $R_{v}^{2}$ & $\operatorname{SEV}(\%)$ & $R_{p}^{2}$ & SEP (\%) & \\
\hline Mean & 0.99 & 0.84 & 0.97 & 1.74 & 0.96 & 2.33 & 6 \\
\hline Maximum & 0.98 & 1.17 & 0.96 & 2.18 & 0.96 & 2.21 & 5 \\
\hline Range & 0.98 & 1.17 & 0.96 & 2.18 & 0.96 & 2.21 & 5 \\
\hline MSC & 0.99 & 1.03 & 0.97 & 1.97 & 0.96 & 2.25 & 5 \\
\hline SNV & 0.99 & 1.05 & 0.97 & 1.99 & 0.96 & 2.29 & 5 \\
\hline $1^{\text {st }}$ derivative & 0.99 & 0.60 & 0.96 & 2.16 & 0.96 & 2.29 & 5 \\
\hline $2^{\text {st }}$ derivative & 0.99 & 0.61 & 0.93 & 2.97 & 0.94 & 3.18 & 5 \\
\hline Raw & 0.99 & 0.61 & 0.97 & 1.90 & 0.96 & 2.22 & 6 \\
\hline
\end{tabular}

* Mean : mean normalization, Maximum : maximum normalization, Range : range normalization, MSC : multiplicative scatter correction, SNV : standard normal variate, $1^{\text {st }}$ derivative : Savitzky-Golay $1^{\text {st }}$ derivative, $2^{\text {st }}$ derivative : SavitzkyGolay $2^{\text {st }}$ derivative, Raw : no preprocessed Raman spectra. 
1. The optimized pretreatment for the PLSR model was the Savitzky-Golay $1^{\text {st }}$ derivative method with five latent variables. The $R_{p}^{2}$ and SEC of the optimal PLSR model were 0.99 and $0.60 \%$ for calibration; the $R_{v}^{2}$ and SEV were 0.96 and $0.411 \%$ for validation; and the $R_{p}^{2}$ and SEP were 0.96 and $2.29 \%$ for prediction, respectively.

The developed PLSR model gave almost similar prediction accuracy and standard error for all of the preprocessing methods. The optimal number factor used in the PLSR model was automatically selected based on the lowest value of the predicted root mean square error (RMSE) in the cross validation process. The plot of the beta coefficient could be compared to Raman bands of specific functional groups to determine which chemicals contributed to unique Raman bands between pure and adulterated onion powder. In Fig. 6, the beta coefficient, which indicates the spectral differences among the samples, showed some important bands representative of the carbohydrates that contributed to the quantitative classification of the pure and adulterated onion powder samples. Fig. 7 shows the actual and predicted concentrations of starch by the PLSR model for the Savitzky-Golay $1^{\text {st }}$ derivative-preprocessed predication set.

\section{CONSLUSIONS}

In this study, Raman spectroscopy was used in conjunction with a multivariate analytical method of PLSR to develop a rapid method for the nondestructive determination of adulteration in powdered food products. A Raman system with $785 \mathrm{~nm}$ excitation was used for adulterated onion powder at all concentration levels from 5\% to $35 \%$. Polynomial curve fitting and seven preprocessing methods were applied to corrected Raman spectra for the optimal PLSR model. The PLSR model developed with Savitzky-Golay $1^{\text {st }}$ derivative-preprocessed Raman spectra showed excellent performance in prediction of added starch concentration in onion powder. This model showed an $R_{c}^{2}$ of 0.99 , SEC of $0.60 \%, R_{v}^{2}$ of $0.96, \mathrm{SEV}$ of $2.16 \%, R_{p}^{2}$ of 0.96 and SEP of $2.29 \%$ using only five latent variables. By interpreting the spectral fingerprinting and beta coefficient, we found that most of the selected wavebands were reflected from starch-related absorptions. The strong Raman bands at 420 and $552 \mathrm{~cm}^{-1}$ in Raman spectra of adulterated onion powder could be attributed to the glucose rings, while the Raman bands at 947, 1366, and $1472 \mathrm{~cm}^{-1}$ could be attributed to the $\mathrm{CH}_{2}$ bending and skeletal vibrations of the $\alpha-1,4$ glycosidic linkage. Thus, the prediction results of the PLSR model were different for various concentrations of cornstarch among adulterated onion powder. The detection limit and sensitivity of conventional Raman spectroscopy were slightly lower than those reported for NIR spectroscopy. However, the Raman technique can be used to measure the constituent parts of spice products without water interference and has the potential for origin based classification of various agricultural products. Therefore, in future studies, we will use Raman spectroscopy method on various powdered food products to detect the authenticity of the product.

\section{ACKNOWLEDGEMENT}

This research was partially supported by Technology Commercialization Support Program, Ministry of Agriculture, Food and Rural Affairs (MAFRA) and by a grant from the Next-Generation BioGreen 21 Program (No. PJ008055), Rural Development Administration, Republic of Korea.

\section{REFERENCES}

Almeida, M. R., R. S. Alves, L. B. L. R. Nascimbem, R. Stephani, R. J. Poppi and L. F. C. de Oliveira. 2010 Determination of amylose content in starch using Raman spectroscopy and multivariate calibration analysis. Anal. Bioanal. Chem. 397: 26932710

Cheng Y. H., S. D. Chen and C. F. Weng. 2006. Investigation of goats' milk adulteration with cows' milk by PCR. Asian-Aust. J. Anim. Sci. 19(10): 1503-1507

Fisk I. D., A. Kettle, S. Hofmeister, A. Virdie and J. S. Kenny. 2012 Discrimination of roast and ground coffee aroma. Flavour $\mathbf{1}$ : 14

Flores G., M. L. R. Castillo, M. Herraiz and G. P. Blanch. 2006 Study of the adulteration of olive oil with hazelnut oil by on-line coupled high performance liquid chromatographic and gas chromatographic analysis of filbertone. Food Chem. 97(4): 742749

Fu, X., M. S. Kim, K. Chao, J. Qin, J. Lim, H. Lee, A. Garrido-Varo, D. Perez-Marin and Y. Ying. 2014 Detection of melamine in milk powders based on NIR hyperspectral imaging and spectral similarity analyses. J. Food Eng. 124: 97-104

Haughey, S. A. S. F. Graham, E. Cancouet and C. T. Elliott. 2012 The application of near infrared reflectance spectroscopy (NIRS) to detect melamine adulteration of soya bean meal. Food chem. 136(3-4): 1557-1561

Hurley I. P., R. C. Coleman, H. E. Ireland and I. H. H. Williams. 2006 Use of sandwich IgG ELISA for the detection and quantification of adulteration of milk and soft cheese. Int. Dairy J. 16: $805-812$

Jha, S. N. and S. Gunasekaran. 2010 Authentication of sweetness of mango juice using fourier transform infrared-attenuated total reflection spectroscopy. J. Food Eng. 101: 337-342

Kizil R., J. Irudayarai and K. Seetharaman. 2002 Characterization of irradiated starches by using FT-Raman and FTIR spectroscopy. J. Agric. Food Chem. 50(14): 3912-3918

Lakshmi, V. 2012. Food adulteration. Int. j. Sci. Inv. Today, 1(2): 101-113

Lee H., B. K. Cho, M. S. Kim, W. H. Lee, J. Tewari, H. Bae, S. I. Sohn and H. Y. Chi. 2013 Prediction of crude protein and oil cotent of soybean using Raman spectroscopy. Sens. Act. B. 185 : 694-700

Lu, C., B. Xiang, G. Hao, J. Xu, Z. Wang and C. Chen. 2009 Rapid detection of melamine in milk powder by near infrared spectroscopy. J. Near Infrared spec. 17: 59-67

Lim J. G., M. S. Kim, I. Back, C. Y. Mo, H. Lee, S. Kang, K. Lee and G. Kim. 2013 Prediction of the melamine particle concentration in milk powder using hyperspectral reflectance imaging and partial least square regression model. Food Eng. Prog. 17(4): 377-386

Qin J., K. Chao and M. S. Kim. 2013 Simultaneous detection of multiple adulteration in dry milk using macro-scale Raman chemical imaging. Food Chem. 138: 998-1007

Rinnan, A., F. V. D. Berg and S. B. Engelsen. 2009 Review of the most common pre-processing techniques for near-infrared spectra. Tr. Anal. Chem. 28(10): 1201-1222

Son H. J., E. J. Hong, S. Ko, J. Y. Choi and B. S. Noh. 2009 Identification of Vegetable Oil-added Sesame Oil by a Mass Spectrometer-based Electronic Nose. Food Eng. Prog. 13(4): 275-281

Tay M., G. Fang, P. L. Chia and S. F. Y. Li. 2013 Rapid screening 
for detection and differentiation of detergent powder adulteration in infant milk formula by LC-MS. Foren. Sci. Int. $232(1-$ 3): $32-39$
Wijaya, W., S. Pang, T. P. Labuza and L. He. 2014 Rapid detection of acetamiprid in food using surface-enhanced Raman spectroscopy (SERS). J. Food Sci. 79(4): 743-747 